\title{
On the Number and Nature of the Solutions of the Apollonian Contact Problem.
}

By R. F. Muirhead, M.A., B.Sc.

The problem of describing a circle to touch three circles, including the nine special cases when one or more of the radii of the given circles are zero or infinite, was solved by Apollonius of Perga in a work which was lost, but of which Pappus has given some account in his Mathematical Collections. Towards the end of the 16th century the problem was again taken up and solved by F. Vieta, and since that time it has formed the subject of investigations by many mathematicians, from many different points of view.

The question as to the number and nature of the solutions in the different cases has not been neglected, and many authors have given more or less extensive tabular summaries of the number of solutions in various cases. One of the most extensive of these tables is that given by L. Gaultier de Tours in his paper in the Journal de l'Ecole Polyt., Calier 16, published in 1813. There he considers the problem of finding a circle to satisfy three given conditions of various sorts. He distinguishes 33 varieties of the problem, as well as 74 varieties of the corresponding problems with reference to the sphere, and tabulates the number of solutions. This table includes the ten cases of the Apollonian problem.

But, as this author points out, the numbers given are only true for certain relations between the data, and it is a matter of difficulty in some cases even to choose the data so that all the theoretical solutions shall be really possible. He makes no attempt to classify the cases according to the relative positions of the given circles, etc., and so far as I know, the only author who has supplied this omission is $O$. Stoll, who has treated the question pretty fully in the Math. Ann., Vol. VI., p. 613, but from the analytical point of view. This paper will be referred to later.

\section{$\$ 1$.}

Consider first the problem of finding the number and nature of the solutions of the Apollonian Problem in the special case when one of the three given circles is a point, and when the contacts with the circles are to be similar, i.e., both external or both internal. 
Let there be two mutually exclusive circles A and B (Fig. 44) and let their external common tangents $t, t^{\prime}$ be drawn. The whole plane is divided into portions $\mathrm{X}, \mathrm{X}, \mathrm{Y}, \mathrm{Y}, Z, Z, \mathrm{~A}, \mathrm{~B}$. Take now a circle having a mutually external contact with $A$ and $B$, and imagine it to pass through all possible positions successively, begining with the tangent line $t$ (which is a circle of infinite radius), and ending with the line $t^{\prime}$ : the whole of the spaces $\mathrm{X}, \mathrm{X}$ will have been swept over once, and the whole of the spaces $\mathrm{Y}, \mathrm{Y}$ twice over ; that is, each point in $\mathrm{Y}$ lies on the variable circle in two distinct positions.

Again, the circle touching $\mathrm{A}$ and $\mathrm{B}$ and containing both, sweeps out $X, X$ once and $Z, Z$ twice over. Note that the points within $A$ and $B$ are not swept over at all, and the points on the circumference are traversed once. Or we may say that as the point moves from without A inwards, the two contact circles which pass through it become coincident when it reaches the circumference, and imaginary when it has passed the circumference.

When the point lies on a common tangent, that tangent itself forms one of the contact circles in question, and may be reckoned as having either external or internal contact with both $\mathbf{A}$ and $\mathbf{B}$.

We can now determine for each given point of the plane the number and nature of the solutions of the problem: To draw a circle through this given point so as to touch the two given circles $A$ and $B$ in the same manner, i.e., both externally or both internally.

We shall adopt a notation for distinguishing the different kinds of contact of the variable circle $K$ with the given circles. We shall denote by $A a$ the contact when $A$ and $K$ are mutually exclusive, by $A \beta$ that when $A$ contains $K$, and by $A \gamma$ that when $K$ contains $A$. Similarly $B a$ means that $B$ touches and excludes $K$, and so on. Again, $A a B a$ or more shortly $\alpha a$ denotes that $K$ touches and excludes both $A$ and $B, a \gamma$ denotes that $K$ excludes $A$ and contains $B$, and so on.

Thus if in Fig. 44 the fixed point $C$ lies in $X$, there are two solutions of our problem, whose nature is indicated by aa and $\gamma \gamma$ : if $\mathrm{C}$ lies in $\mathrm{Y}$ there are two solutions of the nature $a \alpha$; and if in $\mathrm{Z}$, two of the nature $\gamma \gamma$. All this is indicated in the figure.

The solutions of the same problem for the case in which the given circles $A$ and $B$ cut each other are completely indicated in Fig. 45 ; and those for the case in which $A$ contains B, in Fig. 46. 
$\$ 2$.

To treat the corresponding problem when the contacts are dissimilar, we must take the internal instead of the external common tangents. By dissimilar contacts we mean those in which one is external and the other internal, i.e., one is denoted by $a$ and the other by $\beta$ or $\gamma$. The results are shown for the three cases in Figs. 47, 48, 49.

\$3.

We now combine the results of the two previous $\$ S$, and thereby determine the number of solutions of the general problem to find a circle to touch two given circles and a given point, there being no restriction as to the nature of the contact. The resulting diagrams are given in Figs. 50, 51, 52.

Note that when the two given circles cut one another, there are two solutions wherever the given point may be; on the other hand, when $\mathrm{A}$ and $\mathrm{B}$ do not cut each other, there are four solutions or none according to the position of the given point.

$\S 4$.

We proceed to extend the above method to the general Contact Problem of Apollonius, in which there are three circles given, and it is required to find one which will touch all three.

In doing so we use the artifice of "parallel translation of circles."

Take first the case in which $\mathrm{A}, \mathrm{B}$, and $\mathrm{C}$ are the three given circles in decreasing order of size, and where $A$ and $B$ do not intersect, and let us seek the solutions in which the required circle has similar contact with $A$ and with $B$.

Let $A_{0}$ and $A_{1}$ be circles concentric with $A$, having radii equal respectively to the difference, and the sum, of the radii of $A$ and $C$. Similarly for $B_{0}$ and $B_{1}$; and let $C_{0}$ be the centre of the circle $C$. To any circle which touches $\mathrm{A}, \mathrm{B}$, and $\mathrm{C}$ externally, and whose contact is therefore of the nature aaa, there is a corresponding parallel circle passing through $C_{0}$ and touching $A_{0}$ and $B_{0}$ externally. Conversely, for every circle touching $A_{0}$ and $B_{0}$ externally and pass. ing through $\mathrm{C}_{0}$, there is a parallel circle of smaller radius touching 
$A, B$, and $C$ externally. And for every circle touching $A_{1}$ and $B_{1}$ externally and passing through $\mathrm{C}_{0}$, there is a circle touching $A$ and $B$ externally and $\mathrm{C}$ internally, which may be denoted by $a \alpha \gamma$.

The general rule for the nature of the contact of the required circle with $\mathrm{C}$ is that if the radius of the circle passing through $\mathrm{C}_{0}$ has to be increased to get the concentric circle touching $\mathbf{C}$, that circle will contain C. If, however, the radius has to be decreased (algebraically) the contact circle will exclude or be contained by $\mathrm{C}$, according as the radius of the circle passing through $\mathrm{C}_{0}$ is greater or less than that of $\mathrm{C}$.

The results are shown in Fig. 53, and the corresponding results for circles which have dissimilar contact with $A$ and $B$ are shown in Fig. 54. In these figures the dotted lines represent $A_{0} B_{0} A_{1} B_{1}$ and their common tangents, and they are the boundaries of the various portions of the plane considered. The symbol $2 \gamma \gamma \alpha+2 \gamma \gamma \gamma$, for example, indicates that if the centre of $\mathrm{C}$ is in the portion bounded by dotted lines, and thus marked, then there are four circles which will touch $\mathrm{A}, \mathrm{B}$, and $\mathrm{C}$, the contacts with $\mathrm{A}$ and $\mathrm{B}$ being similar-two of these containing $A$ and $B$ and excluding $C$, the remaining two containing all three given circles.

We may note that the ring-shaped spaces between the two circles have two solutions in either of the figures 53,54 , while the external space has four, and the internal spaces none, so that on the whole there will be in this variety of the figure, 8,4 , or 0 solutions of the Apollonian Problem according to the position of $\mathrm{C}$ with reference to $A$ and $D$.

We can obviously interpret the diagram as follows:-If $\mathrm{C}_{0}$ lies in the annulus $A_{1} A_{0}$, it implies that the circles $C$ and $A$ intersect. If $\mathrm{C}_{0}$ lies in the space between two parallel dotted lines then $\mathrm{C}$ cuts the corresponding common tangent to $A$ and $B$. If $\mathrm{C}_{0}$ lies in the dotted rhombus, $\mathrm{C}$ cuts both common tangents, and so on.

It is well known that the contact circles occur in pairs such that each pair has for its radical axis one of the four axes of simili tude of the given circles. It is sometimes assumed that the pairs of circles are such that one touches all 3 circles differently from the other as e.g. when one excludes all three and the other includes them. But it is obvious already that though this is sometimes the case, it happens just as often that one of the pair touches the given circles in exactly the same manner as the other does. 
This has been pointed out by $O$. Stoll in the paper above referred to, as well as the importance of the common tangents in the geometrical interpretation of his results.

Figures 53 and 54 in combination show the number and nature of solutions of the Apollonian Problem for the case in question : but it must be noted that these figures would vary not only in shape but in topologic nature for different relative positions of the circle. Thus the circle $A_{1}$ and $B_{1}$ might intersect, or the exterior centre of similitude of $A_{0}$ and $B_{0}$ might lie without $B_{1}$, etc.

The number of possible different cases of these figures is very great. And if we drop the restrictions that $\mathrm{A}$ does not cut $\mathrm{B}$ and that $C$ is the least of the three circles, the number of different diagrams to which this method would give rise is enormous.

We may however consider the question from a different point of view, from which it will appear that in most of the different cases the number and nature of the solutions can be told almost at a glance by certain simple considerations, leaving a residue to which the foregoing method is more appropriate.

รั5.

The topologically different relative positions of three circles in a plane may be classified as follows into 14 cases, special cases being for the present excluded.

Division I. Circles not intersecting.

$\alpha$. All three circles mutually exclusive.

$\beta$. One contains the other two, which are mutually exclusive.

$\gamma$. One contains another and excludes the third.

s. One " " which contains the third.

Division II. One pair only intersect one another.

u. The third circle excluded by both of these.

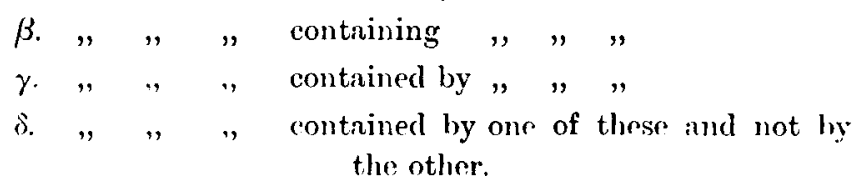


Division III. One pair only do not intersect.

a. This pair mutually exclusive.

$\beta$. One of the pair contains the other.

Division IV. Each circle intersects both the others.

a. No circle contains a crossing point of the other two. (No area common to all.)

$\beta$. One only contains two crossing points of the others. (The area common to all is two-sided.)

$\gamma$. One only does not contain two crossing points of the others. (The common area four-sided.)

$\delta$. Each contains one crossing point of the other two. (The common area three-sided.)

[This classification agrees with that obtained by Stoll from analytical considerations, except that he does not distinguish between the four cases of Division IV., while he distinguishes two cases of III. $\beta$, according to the relative size of the radii. He gives a table showing the number of solutions for each of his cases.]

Now the shapes of the different portions of plane space in these figures are of seven different kinds, which can contain from 0 to 8 contact circles, thus : according as a space (which may or may not extend to infinity) is bounded by one circle, two circles, two circular arcs, three circular arcs, four circular ares, one circle and two arcs, or three circles, the contact circles in it will be $0,0,0,1,2,4$, or 8 in number. This is obvious in the first three cases. It is easily proved in the next three by supposing a circle touching two of the circles to pass successively through all its possible positions within the space in question; and the last number may be arrived at in a similar way, or by the method of $\$ 4$.

Hence we at once arrive at the number, and in most cases the nature of the solutions for any given case. $E . g$. in II. $\beta$ we see there is a quadrangular space which will contain four solutions denoted by $2 \beta a a+2 \beta \gamma \gamma$ (supposing $A$ to be the outer circle); or in IV. $\delta$, there are 8 triangular spaces (including an infinite one) each of which contains one contact circle.

The results of this classification are shown in Table I. 


\section{1}

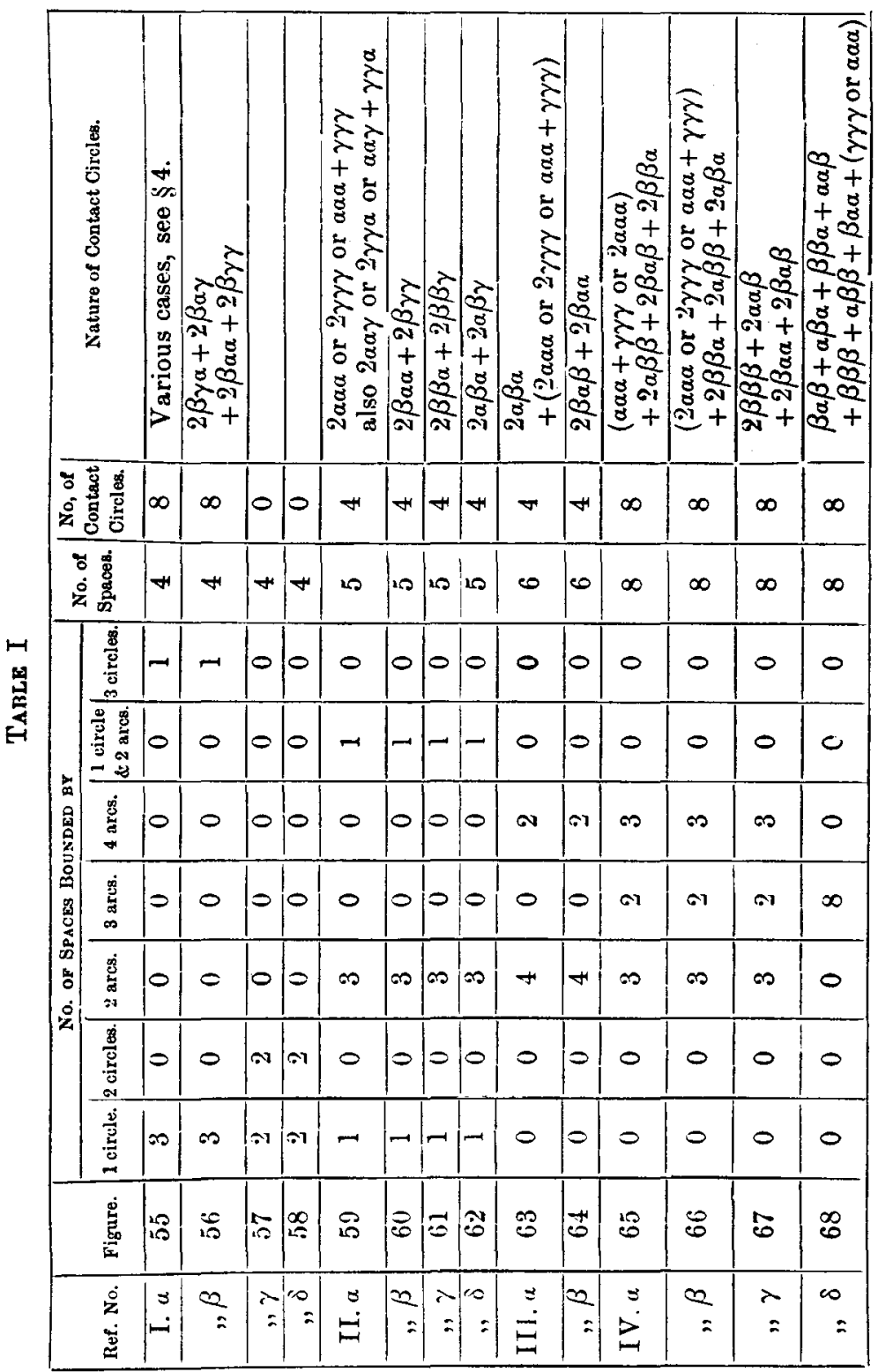

$10 \mathrm{Vol} .14$ 
s 6.

Class A. Two of the given circles touch each other. There are 16 cases which may be classified as A1., A2, or A3., according as the number of points of intersection is 0,2 , or 4 . We shall denote by A1. $a, \beta, \gamma, \delta, \epsilon, \zeta, \eta$, A2. $\alpha, \beta, \gamma, \delta, \epsilon, \mathrm{A} 3 . \alpha, \beta, \gamma, \delta$ the sixteen cases shown in Figures 69 to 85 , respectively.

In general such cases are limiting ones, separating cases of intersecting circles from those which have fewer intersections, or none. Thus A1. $\alpha$ separates cases I. $\alpha$ and II. $\alpha$ of $\$ 5$, and has 6 solutions (the mean of 8 and 4). Two of these are circles which touch the two given touching circles at their point of contact, each representing two solutions in the general case $I$. $\alpha$ which coincide for this limiting case, and disappear in II. $a$. The other four are the same as the solutions of II. $\alpha$.

TABLE II.

\begin{tabular}{|c|c|c|c|}
\hline Reference No. & Figure. & Cases Separated. & $\begin{array}{c}\text { No. of } \\
\text { Solutions. }\end{array}$ \\
\hline A1. $\alpha$ & 69 & I. $a$ and II. $a$ & 6 \\
\hline$\beta$ & 70 & I. $\beta$ and II. $\beta$ & 6 \\
\hline$\gamma$ & 71 & I. $\gamma$ and II $\delta$ & 2 \\
\hline$\delta$ & 72 & I. $\gamma$ and $\mathrm{II}, \alpha$ & 2 \\
\hline$\epsilon$ & 73 & I. $\delta$ and II. $\beta$ & 2 \\
\hline$\zeta$ & 74 & I. $\delta$ and II. $\gamma$ & 2 \\
\hline$\eta$ & 75 & I. $\beta$ and II. $\delta$ & 6 \\
\hline A2. $u$ & 76 & II. $\alpha$ and III. $\alpha$ & 4 \\
\hline$\beta$ & 78 & II. $\delta$ and III. $\beta$ & 4 \\
\hline$\gamma$ & 79 & II. $\delta$ and III. $\alpha$ & 4 \\
\hline$\delta$ & 80 & II. $\gamma$ and III. $\beta$ & 4 \\
\hline$\epsilon$ & 81 & II. $\beta$ and III. $\beta$ & $t$ \\
\hline A3. $a$ & 82 & III. $a$ and IV. $a$ & 6 \\
\hline$\beta$ & 83 & III. $\alpha$ and IV $\beta$ & 6 \\
\hline$\gamma$ & 84 & III. $\beta$ and IV. $\beta$ & 6 \\
\hline$\delta$ & nis & III. $\beta$ and IV. $\gamma$ & i \\
\hline
\end{tabular}


Table II. gives a summary of the corresponding results for this and the other cases of Class $\mathbf{A}$.

One way of tracing the change through a special case from one general case to another, is to treat it from the point of view of $\S 1$, taking for $A$ and $B$ the circles which do not touch

The nature of the solutions in each case can be worked out on the same principles as have been employed above. Or we might deduce them from Table I. For example, Ar $\beta$ separates I. $\beta$ from II. $\beta$. The solutions $2 \beta u \alpha+2 \beta \gamma \gamma$ are common to both, and therefore belong also to $\mathrm{Al} \beta$; while the solutions $2 \beta \gamma \alpha+2 \beta a \gamma$, which exist in I. $\beta$ but not in II. $\beta$, reduce to $\beta \gamma \alpha+\beta a \gamma$ in $A 1 \beta$.

Class B. The three given circles concurrent in one point. Here there are three cases as shown in Figures 86, 87, 88. Each case has 5 solutions, one being a point circle coincident with the point of concurrence, which is the degenerate representative of four circles in the more general case. These three cases are respectively intermediate between IV. $\alpha$ and IV. $\delta$, IV $\beta$ and IV. $\gamma$, and IV. $\beta$ and IV. $\delta$.

Consider now the doubly special cases.

$\$ 7$.

TABLE III.

\begin{tabular}{|c|c|c|c|}
\hline Reference No. & Figure. & Cases Separatel. & $\begin{array}{l}\text { No. of } \\
\text { Solutions. }\end{array}$ \\
\hline C1. $\alpha$ & 89 & Alu and $A 2_{1 \ell}$ & 5 \\
\hline$\beta$ & 10 & $\left\{\begin{array}{r}A l \beta \text { and } A 2 \beta \\
\text { or } A l \eta \text { and } A 2 \epsilon\end{array}\right.$ & $\check{\jmath}$ \\
\hline$\gamma$ & 91 & $A 1 \eta$ and $A 2 \gamma$ & $\overline{5}$ \\
\hline$\delta$ & 92 & $\left\{\begin{array}{r}A l \gamma \text { and } A 2 \alpha \\
\text { or } A l \delta \text { and } A 2 \gamma\end{array}\right.$ & 8 \\
\hline C2. a & $9: i$ & $A \Xi \alpha$ and $A \ddot{\prime} \gamma$ & j) \\
\hline$\beta$ & 94 & $\left\{\begin{array}{r}A 2 \gamma \text { and } A 3 \gamma \\
\text { or } A 2 \beta \text { and } A: \beta\end{array}\right.$ & 5 \\
\hline$\gamma$ & 95 & $A 2 \epsilon$ and $A 3 \gamma$ & $\bar{\jmath}$ \\
\hline
\end{tabular}




\section{4}

Class C. One given circle touches both of the others. I. The other two intersect. 2. They do not.

The various cases are given in Table III.

Note that, as before, the number of solutions is the mean of the numbers corresponding to the two more general cases separated. In each case the circle which touches each of the other circles is itself one of the solutions and really represents two coincident solutions, as also do the other solutions where the circle sought has common contact with two of the given circles. Thus in Figure 96 which indicates the solutions of case C1. $a$, the circles 3, 4 and $B$ each represent two solutions of the more general case I. $a$.

Class D. Combining the conditions of classes A and B. Two cases, shown in Figures 97 and 98 . In either case the number of solutions is 3 , including the point circle coinciding with the point of concurrence, which is the degenerate representative of 6 circles.

Class E. The three given circles concurrent in two different points. One case, Figure 99, where the only two solutions are the two point-circles at the intersections, each of which represents four circles of the general case IV.

Class F. Trẹbly Special Case. The three given circles have a common point of contact. Figures 100, 101. Here the number of solutions is infinite, including every circle which touches them at the common point.

We have now exhausted all the special cases due to coincidence of two or more intersections of two or more out of the three given circles. Another kind of special cases arises when one or more of the given circles is allowed to degenerate into a straight line or a point.

$\$ 8$.

From this point of view there are, as has been observed since the problem was first treated, nine special cases besides the general case of three finite given circles, as shown in Table IV., where the last column gives the greatest possible number of solutions. 
Table IV.

\begin{tabular}{|c|c|c|c|c|}
\hline & $\begin{array}{c}\text { Given } \\
\text { Circles. }\end{array}$ & $\begin{array}{c}\text { Given } \\
\text { Lines. }\end{array}$ & $\begin{array}{c}\text { Given } \\
\text { Points. }\end{array}$ & $\begin{array}{c}\text { Greatest Possible } \\
\text { No. of Solutions. }\end{array}$ \\
\cline { 1 - 2 }$(1)$ & 2 & 1 & 0 & 8 \\
$(2)$ & 1 & 2 & 0 & 8 \\
$(3)$ & 0 & 3 & 0 & 4 \\
$(4)$ & 2 & 0 & 1 & 4 \\
$(5)$ & 1 & 1 & 1 & 4 \\
$(6)$ & 0 & 2 & 1 & 2 \\
$(7)$ & 1 & 0 & 2 & 2 \\
$(8)$ & 0 & 1 & 2 & 2 \\
$(9)$ & 0 & 0 & 3 & 1 \\
\hline
\end{tabular}

Thus the maximum number of solutions is $2^{3-n}$ where $n$ is the number of given points, except in Case (3) where we may say that four of the circles have gone off to infinity, and in Case (6) where in general the lines intersect one another and not the point, so that it belongs to Class II. which has only half the maximum number of solutions of the general case.

All these 9 cases are susceptible of discussion by the method of $\S \varsigma 1,2,3,4$.

If in Fig 44 of $\$ 1$ we imagine the greater circle to become infinite, then the external common tangents become coincident with a line parallel to $A$ touching $B$ on the side remote from $A$. The regions $X X$ disappear, the closed space $Z$ vanishes, and the open space $Z$ goes to infinity, and the whole of the space between $A$ and $B$ belongs to $\mathrm{Y} Y$, so that if $\mathrm{C}$ lies in this region, the solutions are denoted by $2 a \alpha$, while if $\mathrm{C}$ lies elsewhere there is no solution.

Taking the corresponding case of $\$ 2$, viz., Fig. 47, we see that a similar result comes out, the solutions for the space between $A$ and $B$ being denoted by $2 \alpha \gamma$, i.e., there are two contact circles containing B. Thus in Case (5) when the line and circle do not intersect, there are two contact circles which exclude $B$, and two which contain $B$. 
Note that in treating a line as a circle of infinite radius we may arbitrarily choose either side of the line to be the interior: but in order to apply the previous results, we must consistently keep to the choice made.

We might arrive at the results just found more directly, by observing that the variable circle which touches $A$ and $B$ and excludes $B$ passes over every point of the region between the two twice over, as also does the variable contact circle which contains $B$.

Taking cases (1) to (9) seriatim :-

In (1) we note that the various cases are as a rule special cases separating one case of Table I. from another, though some of them are special forms of a single case of Table $I$. The results are shown in Table $\mathrm{V}$.

TABLE V.

\begin{tabular}{|c|c|c|c|}
\hline Figure. & $\begin{array}{l}\text { Cases of Table I. } \\
\text { Separated. }\end{array}$ & $\begin{array}{c}\begin{array}{c}\text { No. of } \\
\text { Solutions. }\end{array}\end{array}$ & Nature of Solutions. \\
\hline 102 & I. $\alpha, \beta$ & 8 & $2 \alpha \gamma+2 \gamma \alpha+2 \gamma \gamma+2 \alpha \alpha$ \\
\hline 103 & I. $\gamma$ & 0 & \\
\hline 104 & I. $\gamma, \delta$ & 0 & \\
\hline 105 & II. $a, \delta$ & 4 & $2 \alpha \alpha+2 \alpha \gamma$ \\
\hline 106 & II. $\alpha, \beta$ & 4 & $2 \mu \alpha+2 \gamma \gamma$ \\
\hline 107 & II. $\gamma, \delta$ & 4 & $2 \beta a+2 \beta \gamma$ \\
\hline 108 & III. $a$ & 4 & $4 \alpha \alpha$ \\
\hline 109 & III. $\beta$ & 4 & $4 \beta a$ \\
\hline 110 & III. $\alpha, \beta$ & 4 & $2 \alpha \alpha+2 \beta a$ \\
\hline 111 & IV. $\alpha, \beta$ & 8 & $2 u \beta+2 \beta u+4 u s$ \\
\hline 112 & IV. $\delta$ & 8 & $2 \alpha a+2 \alpha \beta+2 \beta a+2 \beta \beta$ \\
\hline 113 & IV. $\beta, \gamma$ & 8 & $2 u \alpha+4 u \beta+2 \beta \beta$ \\
\hline
\end{tabular}


Case (2). Here I. can only exist in a sub-case, i.e., when the lines are parallel. There are 4 solutions if we have II. (or III.). i.e., if the lines are not both cut by the given circle, and 8 solutions if we have IV., i.e., if both lines cut the given circle. The nature of the contacts is obvious in the several varieties of this case.

Case (3). This belongs in general to IV., but four of the circles have gone off to infinity, leaving only four solutions, the in- and escribed circles of the triangle.

Case (4). This has already been treated in $\S \S 1,2,3$.

Case (5) When the line and circle do not intersect, we have as at the beginning of this $\S$, four solutions $(2 \mu+2 \gamma)$ or none; and when the line and circle do intersect, two solutions $(2 \alpha$ or $2 \beta$ according as the point is without or within the given circle).

Case (7). We might treat this by $\$ \S 1,2$, taking $B$ to be a point-circle, in which case the interior region $Z$ vanishes, as in Fig. 114. Thus if $\mathrm{C}$ lies in 7 , there are two solutions $(2 \alpha)$; if in $Z$, $2 \gamma$; if in $X, a+\gamma$. In other words there are two exterior contact circles if $\mathrm{A}$ is met by $\mathrm{BC}$ produced but not by $\mathrm{BC}$, two interior contacts if $\mathrm{BC}$ cuts $\mathrm{A}$ twice, and one of each if neither $\mathrm{BC}$ nor $\mathrm{BC}$ produced cuts $\mathrm{A}$, and none if the circumference of $\mathrm{A}$ separates $\mathrm{B}$ and $\mathrm{C}$.

The same results could be got by considering the variable circle passing through B and $\mathrm{C}$.

Cases (6), (8), (9) are all obvious.

Each problem of $\$ 8$ might be considered under the special conditions of Classes $\mathrm{A}$ to $\mathrm{F}$ and thus give rise to yet more special cases; and there would be no difficulty in applying the same principles as we have used above to determine the number and nature of their solutions.

There are also many other kinds of special cases, e.g., when intersecting lines become parallel, etc.

On the other hand the above methods may be applied to the corresponding Contact Problem for Spheres instead of Circles. In place of the common tangent lines of two circles, of $\$ \$ 1,2$ we should have to consider the common lunyent planes of three splheres. 